\title{
Indigenous Knowledge Systems for Local Weather Predictions: A Case of Mukonchi Chiefdom in Zambia
}

\author{
Mabvuto Mbewe ${ }^{1}$, A. Phiri ${ }^{2} \&$ N. Siyambango ${ }^{3}$ \\ ${ }^{1}$ Mulungushi University, School of Agriculture and Natural Resources, Kabwe, Zambia \\ ${ }^{2}$ Mulungushi University, Directorate of Research and Post Graduate Studies, Kabwe, Zambia \\ ${ }^{3}$ University of Namibia, Multidisciplinary Research Centre, Windhoek, Namibia \\ Correspondence: N. Siyambango, University of Namibia, Multidisciplinary Research Centre, Private Bag 13301, \\ Windhoek, Namibia. E-mail: nsiyambango@unam.na; nguzasiya@gmail.com
}

Received: February 2, 2019

Accepted: February 26, 2019 Online Published: March 30, 2019

doi:10.5539/enrr.v9n2p16

URL: https://doi.org/10.5539/enrr.v9n2p16

\begin{abstract}
The purpose of the study was to unravel constituents of the indigenous knowledge systems (IKS) and appreciate people's experiences in predicting the weather in daily undertakings. The objectives of the study were; to identify factors or systems used, establish the knowledge used in predicting the weather and compare the indigenous and current scientific method of predicting the weather. Qualitative and quantitative research designs were used. Primary data was collected through semi structured, face-to-face and in-depth interviews. This was complemented by secondary data collected through desk reviews of relevant published materials. The findings reveal that indigenous knowledge systems have been employed by people of Mukonchi chiefdom since time immemorial. There has also been reliance on IKS to make decisions pertaining to livelihoods such as agricultural activities. However, IKS in the area remains undocumented. Observation of several occurrences in combination or singularly relating to plants, animals, insects and astronomical events were factors of significant importance in the knowledge of weather extrapolation. Elements such as age, frequency of use of the IKS and level of education were seen to be of momentous prominence in utilisation of the indigenous knowledge as modern means of weather forecasting which are applicable to local community environment.
\end{abstract}

Keywords: Indigenous, Weather Knowledge, Climate Systems, Forecasting

\section{Introduction}

Before the modern meteorological instruments, many traditional societies in Africa, and we believe elsewhere, depended on the indigenous knowledge systems (IKS) to forecast the weather (Risiro et al., 2012; Briggs, 2005). Local communities exposed to disaster risks utilised IKS as the basis for their decision-making at micro, messo and macro levels in managing their livelihoods at local community level (Phiri et al., 2019). In general, IKS was specific and unique to a particular culture. It consisted of various features such as historical experiences, plants, insects, genetics, relationships and rituals. IKS was extremely useful and important in decision-making not only in planning but conservational activities, as well as in averting life threatening weather related events. However, although most local communities relied on IKS to understand weather and climate patterns, the extent to which indigenous knowledge was used in forecasting weather in Mukonchi chiefdom has not been documented. In fact it has been declining and in danger of being lost. This is because IKS exists in oral tradition and is passed on from generation to the other. So, as the elderly who possess this knowledge pass on fewer people remain with the knowledge and consequently its usage. Factors that influence the use of IKS to predict weather have not been adequately studied. On the other hand, there have been advances in climate modelling resulting into increased ability to predict rainfall in many parts of the world by using dynamical forecasts or statistical methods. Despite these advances, the information has not reached majority of the villagers and not well documented to enhance early warning capabilities to suit subsistence level needs. This has been coupled with lack of understanding and ownership of these scientific methods by local farmers and communities (Patt, 2002). Based on the findings, the discussion therefore intends to elucidate the critical nature of the IKS in people's lives in traditional settings as regards weather forecasting and how it can be integrated into communal living experiences. It is believed that IKS can add value to current weather forecasting and therefore enhance human living conditions by providing safeguards to reduce vulnerabilities from weather excess. As the findings indicate, IKS was embedded in 
Mukonchi community practices, institutions and sectors including agriculture, health, and natural resource management. IKS was pervasive in people's lives as tacit knowledge that was not easily modifiable but provided local basis for problem-solving strategies. But IKS was context specific which then allowed for the flourishing of the local situation and provision of enabling environment for activities designed to help the communities bring about development (World Bank Group, 2011). It is hoped that the discussion, based on the study findings, can provide the opportunity of how to integrate IKS into agricultural life sustaining activities to reduce weather vagaries and excesses.

Over time, the local communities in the chiefdom have developed the art of assessing weather conditions using their experience, observation and cumulative knowledge from generations. It is largely based on keen observation of various systems including nature of clouds, faunal, floral, sun, moon, wind, migration patterns of birds and other physical changes in their surroundings that precede or accompany meteorological phenomenon of larger interest (Rautela et al., 2015). In most local communities in the chiefdom, the inhabitants have tended to rely on and extensively apply IKS to determine the weather. Even at this stage of development, many parts of Zambia including the Munkochi chiefdom do not have access to modern equipment that can provide specific, dependable, and appropriate information services for use by the farmers, fishermen, pastoralist and others in their varied life situations. Such useful information is fairly limited to those with modern facilities such as print and electronic media. Besides acquisitions of such facilities there are issues of accessibility and affordability among villagers. In addition, issues of overwhelming poverty and illiteracy exacerbate the challenges of accessibility, affordability and maintenance.

The application of the indigenous knowledge was not scientific per se because by its nature is largely based on intuition and history. The accumulation of knowledge which is acquired through daily observation (Ngulube, 2017) is critical and essential. This knowledge, it must be emphasized, is not depended on scientific observation and processes. Over time, the knowledge becomes considerable and turns into experience. This knowledge is built upon faith and intuition. It is different from the scientific type which provides an explanation based on the data from which extrapolations are made. By the way, according to the indigenous knowledge, the explanations of events or phenomenon is context specific in terms of space and time. It is therefore argued that predicting of weather in traditional societies such as Munkonchi is an art (Ngulube, 2017). People regard the practice of social work being both scientific and as an art. While much of the practice is scientifically based in terms of knowledge drawn from various disciplines, the practice of social work is partly based on intuition and practice wisdom which come after a long span of practice. Similarly, weather prediction based on the indigenous systems is an art.

\section{Indigenous Knowledge System versus Conventional Forecast}

In Zambia, many communities have used indigenous knowledge systems (IKS) as a critical knowledge base and survival tool for adapting to extreme climate events and other natural hazards. Disasters affect people at different levels based on their capacity and vulnerability to withstand them (Phiri et al., 2016). They have developed techniques and strategies for forecasting weather events, and managing climate variability including coping mechanisms to respond to harsh conditions of the local environments (Ngulube, 2017). The indicators for weather prediction do not generally differ among communities. They are broadly classified as meteorological (moon and sun), biological (treeflowering and fruiting, leaf burst, bird and insect movement andbehaviour), geographical (temperature extremes and wind movements) and some bird types are common indicators for short-term forecasting (Cruz, 2007). Traditionally, farmers have also based their decisions about crops and irrigation cycles on indigenous knowledge for weather prediction. However, in the recent times, the weather patterns in Zambia have drastically changed and have posed a challenge on the IKS knowledge and weather prediction. For example, Zambia has in the recent past experienced both el-Niño and el-Nina weather phenomena (Goddard \& Dilley, 2005). These are extreme weather phenomena affecting both macro and micro factors that have negatively impacted livelihoods and have outpaced the IKS ability to predict the weather and its effects. These are premised on scientific or conventional knowledge. These extreme weather patterns could be responsible for events such as army worms that wipe the entire crops leaving people with nothing to live on.

\section{Indigenous Knowledge Systems and Climate System in Munkochi Chiefdom}

Munkonchi Chiefdom, the study area, is located in the Southern province of Zambia. It is one of the 10 provinces of Zambia and comprises of 11 administrative districts. According to the 2010 Census of Population and Housing, the Province has a total population of 1,589,926 with 1,197,751 people residing in rural areas. The population in the province therefore is predominantly rural and the people are generally referred to as Tonga speaking (2010, Census of Population and Housing). They generally rely on the cultivation of land and 
production of crops such as maize, cotton, sweet potatoes, beans and other major crops. They are also involved in animal husbandry especially cattle farming as a source of livelihood and a significant socio-economic activity. In addition, the people of southern province and Munkonchi in particular are also involved in fishing as another source of livelihood but within the context of rural economy. These and several other activities are important not only as livelihoods but as sources of household income, rural economy, maintenance activities of day-to-day human life and as part of the core economic activities of the Mukonchi chiefdom.

Notably, the wet seasons in Zambia have become predominantly characterized by late onset of rains, shorter rainy seasons, skewed rainfall distribution, raging fires and intenserainstorms are more frequent. Drought occurs more frequently and uncharacteristically with long dry spells. This has raised questions over the reliability of indigenous knowledge to forecast weather (Stenek et al., 2011). Obviously, the increase in climate variability and the climate change, characterised by increased frequency and severity of extreme climatic events, pose a serious threat to the livelihood of most people not only in Zambia but elsewhere in Africa and beyond. The adverse impacts of climate variability and climate change on various socio-economic sectors, environment and livelihoods are already vivid and have the potential to undermine or reverse the progress so far attained in the development of the socio-economic well-being of Zambians (Nindi et al., 2011). Hence, the need to adopt sound strategies that can produce increased accuracy, reliability and dependability in weather and climate predictions. However, utilization of these strategies will require training of the rural people so that they can adopt them for use. This will enable most rural communities to make informed, timely and effective decision for their farming activities leading to increased productivity and survival (Risiro et al., 2012).

\section{Research Methodology}

The aim of the study was to investigate how prediction of the weather was carried out by the use of IKS and how the information, in various forms, was applied by the people in traditional settings. The study adopted a mixed methods approach which is a combination ofquantitative and qualitative data and exploratory and descriptive designs which were considered appropriate because they allowed for more flexible strategies of data collection to answer the research questions (Masahiro, 2017). In addition, these approaches accommodated the qualitative method which was also considered appropriate to reveal experiences of the respondents in the study and especially that the information sought was rather intuitive and based on memory. It was therefore, important to obtain detailed information in order to decipher and understand the experiential details about weather prediction.

The study used the qualitative research method to understand the knowledge base and experiences of how to predict the weather using the IKS in Munkochi chiefdom. A face-to-face, in-depth unstructured interviews were conducted to appreciate people's experiences and personal interpretation of weather prediction. This approach was suitable since it provided more latitude to probe especially that little is known about weather prediction in traditional societies in general and in particular in Munkochi area. In addition the key informants were also included in the interviews. Tonga language was used in the interviews since the respondents were conversant with the language. The idea was to allow them express themselves freely. The interviews were audio taped and later transcribed.

The population for the study was purposively drawn from the residents of Munkochi area who have lived in the place for forty years and above. This was based on the assumption that they have acquired sufficient knowledge of IKS and the cultural nuisances of weather prediction. However, it is acknowledged here that purposive sampling may have elements of subjectivity because selection of respondents depended on the researcher. The sample size comprised of 96 respondents categorised into age groups of: 40-49, 50-59 and 60 and above including 5 key informants (4 elders above 60 years and a village headman). Variables employed were age, gender, level of education, period of residence, frequency use, source of IKS, and type of IKS. It was assumed that respondents of 40 and above had used, acquired sufficient IKS and experience for predicting weather events over the years. In addition, the primary data was complimented by the secondary data which was derived from published materials, official documents, annual reports, commissioned research and relevant literature on weather prediction from various experience.

\section{Presentation and Discussion of the Findings}

Data collected was coded and analysed using the Statistical Package for Social Sciences (SPSS) and Excel package. The data was presented in form of graphs and bar charts to describe the demographic information which included age, period of residence, level of education, frequency of use of IKS and challenges in using IKS Chi square tests were also performed to test the relationship between Social Economic variables and choice of IKS signs/indicators in using IKS amongst the study respondents. 


\section{Identifying IKS Signs/Indicators Used to Predict Weather in Mukonchi}

This section outlined the findings of this study on the types of IKS signs/indicators commonly used in Mukonchi. It also categorized these IKS signs/indicators into biological, geographical and meteorological category type. The study also outlined the frequency of use as well as the reliability of some of the common IKS signs/indicators in Mukonchi. Cross tabulations and chi square tests at 90 per cent level of confidence were used to ascertain relationships of operational variables and outcome.

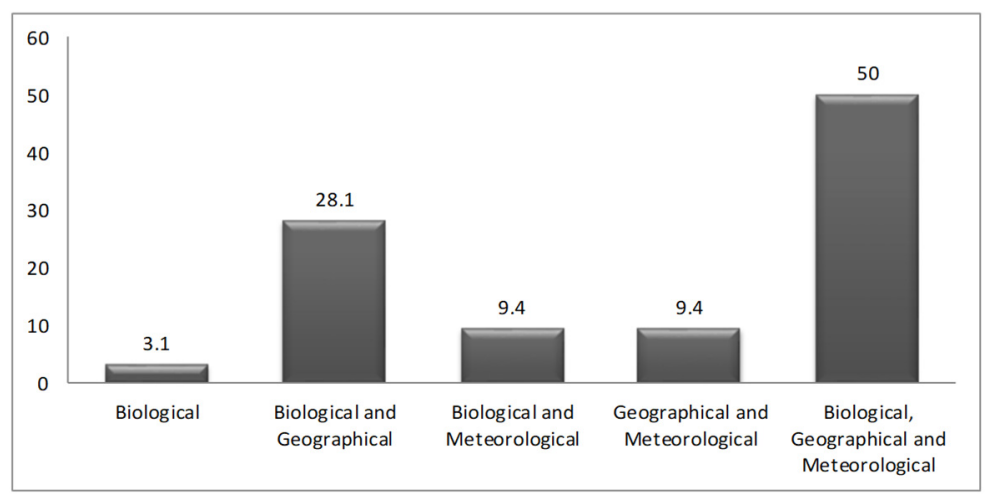

Figure 1. Type of IKS signs/indicators commonly used in Mukonchi

Figure 1 above shows IKS signs/indicators commonly used by respondents to predict weather in Mukonchi. These were categorised as biological, geographical and meteorological signs/indicators. $3.1 \%$ of the respondents used biological signs/indicators to predict weather events. $28.1 \%$ used Biological and Geographical signs/indicators with 9.4\% using Biological and Meteorological signs/indicators or Meteorological and geographical signs/indicators whilst $50 \%$ of the respondents used Biological, Geographical and Meteorological signs/indicators. These, without doubt, shows Biological signs/indicators are least preferred at $3.1 \%$ compared to the Biological, Meteorological and Geographical signs/indicators at 50\% indicating most preference. This is a significant finding in terms of utilisation and integration or mainstreaming of IKS into weather forecasting in the rural settings. They also indicate the kind of knowledge systems that were used in the process of application.

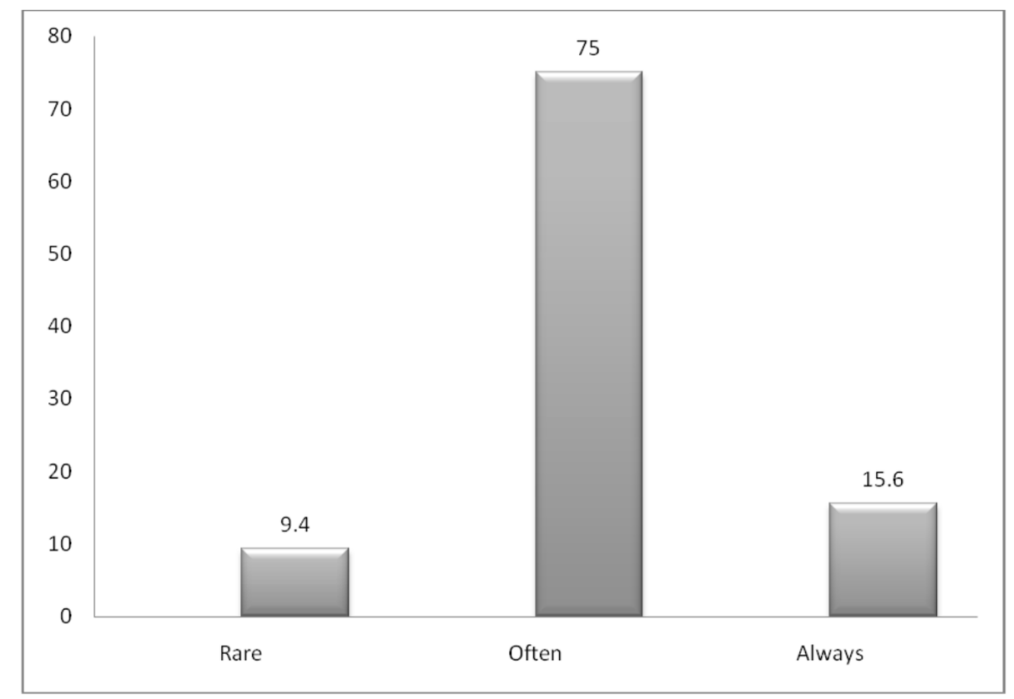

Figure 2. Frequency of use of IKS (\%)

Figure 2 above shows the frequency of use by the respondents of IKS signs/indicators to predict weather. From the findings, it emerged that $9.4 \%$ of the respondents rarely used IKS to predict weather events but $75 \%$ of the respondents often used IKS signs/indicators to predict weather and $15.6 \%$ always used IKS to predict weather. What is important here is the fact that all the respondents acknowledged use of IKS but the difference lies in the 
frequency. It was observed that local people in Mukonchi mostly relied much on forecast information from IKS than from conventional method. Perhaps most importantly the data have clearly demonstratedthat IKS is quite popular at least at the time of the study because it wasused by the majority of the people in Munkochi area.

Based on the findings (Table 1), the respondents used tree fruiting and flowering, insect movement and behaviour (biological); temperature extremes and wind movement (geographical); moon and sun (meteorological) as signs/indicators for weather forecasting. Plant species were mentioned to be handy in weather predictions in a variety of ways. Some plants changed their morphology with season, plant species such as Brachystegia and Julbernardia bloom into green and release tender leaves markingthe onset of the rain season. These trees start to bloom as from September and shed offtheir leaves during the dry season. Abundance of Parinari (Mupundu) fruits indicated poor rains or drought in that year while abundance of Uapaka (Musuku) fruits indicated good rains. Other biological indicators included abundance of mushrooms as an indication of good rainfall distribution for that year. Insects (Nakapelele) appeared in the rainy season (October-November) only and some insects like ants and Ifiyongoli could only be seen in rainy season (October-March) or when the rainy season was approaching (October-November). When there were a lot of caterpillars (ifishimu) then there was likely to be poor distribution of rains or delayed onset of rain (Centre for International Studies and cooperation, 2011). This was also the case in a similar study in Zimbabwe were IK signs/indicators were broadly classified as biological, geographical and meteorological (Risiro et al., 2012).

Table 1. IKS signs/indicators and type: IKS signs/indicators (short term and long term) and their type were shown in table 1 below

\begin{tabular}{|c|c|c|}
\hline INDICATOR & INTERPRETATION & TYPE \\
\hline$\underline{\text { Short term }}$ & Then expect rains within an hour. & Geographical \\
\hline \multicolumn{3}{|l|}{ If gentle winds blow from West to East. } \\
\hline High temperatures during the day in the rain season. & Then expect rains that day. & Geographical \\
\hline Moon is full and shinning. & Means there will be no rains for days. & Meteorological \\
\hline When there are strong winds blowing from East to West. & Then it will rain. & Geographical \\
\hline Lightening, thunder and cloud cover. & Then expect rains within an hour. & Meteorological \\
\hline Moon appears in a clear sky. & Then there won't be rains. & Meteorological \\
\hline $\begin{array}{l}\text { When moon settles in the dark clouds and moves with } \\
\text { the dark clouds. }\end{array}$ & Then it will definitely rain. & \\
\hline$\underline{\text { Long term }}$ & Then there is likely to be poor distribution of rains or delayed & Biological \\
\hline When there are a lot of caterpillars (ifishimu). & onset of rains. & \\
\hline Abundance of Parinari(Mupundu) fruits. & Indicate poor rains or drought in that year. & Biological \\
\hline Abundance of Uapaka(Musuku) fruits. & Indicate good rains in that year. & \\
\hline Abundance of mushrooms. & Indicate good rainfall distribution for that year. & Biological \\
\hline Insects (Nakapelele). & Appear in the rainy season only. & Biological \\
\hline Some particular birds & $\begin{array}{l}\text { Only migrate to a place where the onset on rainy season is } \\
\text { close. }\end{array}$ & Biological \\
\hline Certain types of birds (Koloka) make distinctive sounds. & That signifies onset on rain season. & Biological \\
\hline If a crow (Mwankole) still has eggs in its nest. & Then rains won't start until the eggs hatch. & Biological \\
\hline If it is very cold in June. & Then there would be a lot of rain that year & Geographical \\
\hline Some insects like ants and Ifiyongoli & $\begin{array}{l}\text { Can only be seen in rainy season or when the rainy season is } \\
\text { approaching }\end{array}$ & Biological \\
\hline
\end{tabular}

Source: (Mabvuto et al., 2016).

If gentle winds blow from West to East, then rain was expected within an hour. High temperatures during the day in the rain season (October-March) were an indication of impending rains that day. When there were strong winds blowing from East to West then it would not rain. These were categorised under geographical indicators. On the other hand, a full moon shinning meant no rains for days. Lightening, thunder and cloud cover (Geographical) was an indication of rains about to fall. If the moon appeared in a clear sky then there would be no rains, but when it settled in the dark clouds and moves with the dark clouds then it would definitely rain. These indicators were categorised under meteorological methods (Makwara, 2013).

Table 1 further establishes that there are IKS signs/indicators that respondents used for short term and long term forecasts. This was also the case in a similar research carried out in India where indicators were categorised in 
terms of short and long term indicators. Short term indicators could be used to predict rainfall within 24 hours and 2-10 day period. Long term or seasonal indicators were used to predict weather in months before the events could unfold (Amanor, 1993).

\subsection{Factors in the Use of IKS to Predict Weather}

This section outlines some of the social factors that influence the respondents' knowledge in the use of IKS to predict weather. Cross tabulations and chi square tests at 90 per cent level of confidence were used to ascertain relationships of operational variables and outcome.

Table 2. Factors that influence IKS

\begin{tabular}{|c|c|c|c|c|c|c|}
\hline \multirow[t]{2}{*}{ Characteristic } & \multicolumn{4}{|c|}{ Perception on use of IKS } & \multirow{2}{*}{$\begin{array}{l}\text { Total } \\
\% \\
\end{array}$} & \multirow[t]{2}{*}{ P-Value } \\
\hline & Very difficult & Difficult & Easy & Very easy & & \\
\hline \multicolumn{7}{|l|}{ Age } \\
\hline $40-49$ & $4.5 \%$ & $40.9 \%$ & $50.0 \%$ & $4.5 \%$ & 100 & \multirow{3}{*}{$0.000 * * *$} \\
\hline $50-59$ & $0.0 \%$ & $28.6 \%$ & $14.3 \%$ & $57.1 \%$ & 100 & \\
\hline $60-69$ & $0.0 \%$ & $33.3 \%$ & $66.7 \%$ & $0.0 \%$ & 100 & \\
\hline \multicolumn{7}{|l|}{ Gender } \\
\hline Male & $5.9 \%$ & $29.4 \%$ & $35.3 \%$ & $29.4 \%$ & 100 & \multirow{2}{*}{$0.000 * * *$} \\
\hline Female & $0.0 \%$ & $46.7 \%$ & $53.3 \%$ & $0.0 \%$ & 100 & \\
\hline \multicolumn{7}{|c|}{ Level of education } \\
\hline Primary & $10.0 \%$ & $60.0 \%$ & $30.0 \%$ & $0.0 \%$ & 100 & \multirow{3}{*}{$0.000 * * *$} \\
\hline Secondary & $0.0 \%$ & $25.0 \%$ & $55.0 \%$ & $20.0 \%$ & 100 & \\
\hline College & $0.0 \%$ & $50.0 \%$ & $0.0 \%$ & $50.0 \%$ & 100 & \\
\hline \multicolumn{7}{|c|}{ Period of residence } \\
\hline $0-10$ & $5.6 \%$ & $50.0 \%$ & $44.4 \%$ & $0.0 \%$ & 100 & \multirow{3}{*}{$0.000 * * *$} \\
\hline $11-20$ & $0.0 \%$ & $50.0 \%$ & $50.0 \%$ & $0.0 \%$ & 100 & \\
\hline $21-30$ & $0.0 \%$ & $16.7 \%$ & $41.7 \%$ & $41.7 \%$ & 100 & \\
\hline \multicolumn{7}{|c|}{ Frequency of use of IKS } \\
\hline Rare & $33.3 \%$ & $33.3 \%$ & $33.3 \%$ & $0.0 \%$ & 100 & \multirow{3}{*}{$0.000 * * *$} \\
\hline Often & $0.0 \%$ & $41.7 \%$ & $45.8 \%$ & $12.5 \%$ & 100 & \\
\hline Always & $0.0 \%$ & $20.0 \%$ & $40.0 \%$ & $40.0 \%$ & 100 & \\
\hline \multicolumn{7}{|l|}{ Source of IKS } \\
\hline Family & $0.0 \%$ & $31.2 \%$ & $43.8 \%$ & $25.0 \%$ & 100 & \multirow{3}{*}{$0.013 * * *$} \\
\hline Community & $10.0 \%$ & $50.0 \%$ & $40.0 \%$ & $0.0 \%$ & 100 & \\
\hline Oracles & $0.0 \%$ & $33.3 \%$ & $50.0 \%$ & $16.7 \%$ & 100 & \\
\hline \multicolumn{7}{|l|}{ Type of IKS } \\
\hline Bio & $0.0 \%$ & $100 \%$ & $0.0 \%$ & $0.0 \%$ & 100 & \multirow{5}{*}{$0.000 * * *$} \\
\hline Bio \& Geo & $0.0 \%$ & $44.4 \%$ & $44.4 \%$ & 11.1 & 100 & \\
\hline Bio \& Met & $0.0 \%$ & $0.0 \%$ & $100 \%$ & $0.0 \%$ & 100 & \\
\hline Geo \& Met & $33.3 \%$ & $33.3 \%$ & $33.3 \%$ & 0.0 & 100 & \\
\hline Bio, Geo \& Met & $0.0 \%$ & $37.5 \%$ & $37.5 \%$ & $25.0 \%$ & 100 & \\
\hline \multicolumn{7}{|c|}{ Confidence in using IKS } \\
\hline Not confident & $0.0 \%$ & $66.7 \%$ & $33.3 \%$ & $0.0 \%$ & 100 & \multirow{3}{*}{$0.000 * * *$} \\
\hline Indifferent & $8.3 \%$ & $66.7 \%$ & $25.0 \%$ & $0.0 \%$ & 100 & \\
\hline Confident & $0.0 \%$ & $11.8 \%$ & $58.8 \%$ & $29.4 \%$ & 100 & \\
\hline
\end{tabular}

Table 2 above ascertained whether there was a relationship between respondents' challenges in using IKS in relation to:
a) Age
b) Gender
c) Level of education
d) Period of residence in Mukonchi
e) Frequency of use of IKS 


\section{f) Source of IKS}

\section{g) Type of IKS}

h) Confidence level in using IKS

With chi square at 90 per cent confidence level, the study found that there was a significant difference between the respondents' perception on the use of IKS to predict weather with the social factors like age, sex, level of education and period of residence. There was a very high significant difference between the challenges using IKS to predict weather with the respondents' frequency of use of IKS, source of IKS, type of IKS signs/indicators and confidence level in using IKS.

\subsection{Social Factors}

In terms of social factors that influence people's perception in the use of IKS to predict the weather, the study findings demonstrate that there was a significant relationship between the respondents' age group and the respondents' perception in the use of IKS to predict weather. It was found that respondents in the higher age groups had less challenges using IKS to predict weather than those in lower age groups. In other words the younger respondents had difficulties in predicting weather i.e. $40.9 \%$ of respondents aged between $40-49$ years said it was difficulty to used IKS to predict weather while $50 \%$ found it easy. $57.1 \%$ of those aged between 50-59 years said it was 'Very easy' to use IKS to predict weather with $28.6 \%$ saying it was difficulty. But more than the majority of the respondents in the age group 60-69 years $(66.7 \%)$ said it was 'Easy' to use IKS to predict weather events with $33.3 \%$ saying it was difficulty. The understanding confirms the age variable as being significant in the weather prediction. This relates to the amount of knowledge accumulation as one advances in age. Consequently, the older generation becomes the repository of IKS. But the danger is that as their memory weakens or pass on, IKS is lost and hence the need to document.

The study findings also showed that there was significant correlation between respondents' level of education and their perception in the use of IKS to predict weather. It was found that respondents' with higher education level faced less challenges than those with lower education levels in the utilisation of IKS. Similarly, a study in Nigeria showed that local farmers with some education background were able to use IKS to predict weather systems such as rainfall, sunshine, thunderstorms, windstorms and harmattan (a dry, dusty wind that blows along the north-west coast of Africa) to prepare for future agricultural activities to enhance their livelihoods (Ajibade, 2003). While there could be other factors, the subjects taught in schools which have a component of weather studies like geography might bear some influence in the understanding and interpretation of weather factors. This makes it easier for farmers with such knowledge to employ IKS which may not easily be the case for those without education. These findings therefore tend to support the study undertaken in Zimbabwe (Boko et al., 2007) that concluded that farmers' willingness to use conventional scientific climate forecasts together with IKS increased with education. Therefore, the more education available among the end users the easier it is to integrated both conventional scientific systems and IKS. This is therefore a notable finding in the process of indigenization of the weather prediction and making it more widely available in rural areas. Methods of community mobilisation and participatory research can be used as tools to engage communities in integrating both IKS and conventional weather forecasting. It is something that must be stimulated by the people themselves in a participatory fashion, sustained in order to improve the socio-economic well-being of the people. There is need to advocate for inclusion or integration of IKS in the meteorological training and activities.

Regarding the period of residence in Mukonchi and acquisition of knowledge in weather determination through the use of IKS, the study found that respondents who had lived in Mukonchiarea for a longer period of time faced less difficulties in using IKS than those who had lived there for a shorter period. This may be so because indigenous knowledge has been defined as knowledge that is unique to a particular culture and society (Ngulube, 2017). It is generally assumed that the longer one lives in the culture the more knowledge one accumulates. Or the older one is the more knowledgeable she or he is about the culture. The knowledge is the basis for decision-making in life tasks such as agriculture, health, natural resource management and other activities. IKS is embedded in community practices, institutions, relationships and rituals. It is essentially tacit knowledge that is not easily modifiable (Ngulube, 2017). So, the longer one lives in Mukonchiarea the more one accumulates knowledge about the IKS and weather forecasting.

The acquisition of knowledge of IKS gives people ability and confidence to predict the weather with ease. In fact the more they use IKS to predict the weather the more they grow in knowledge and confidence and the more they acquire the IKS. So one builds the repository of knowledge by frequent use of the IKS. This actually testifies to the old adage: "practice makes perfect". In fact this finding echoes similar findings that was undertaken in Burkina Faso where farmers' forecasting knowledge exponentially grew with the use of the 
knowledge and techniques (Roncoli et al., 2001). Those who used the methods more grew in confidence and faced less challenges.

\subsection{Comparing the IKS and Conventional Weather Forecasting Methods}

Table 3. Comparison between IKS and Coversional weather forecast

\begin{tabular}{ll}
\hline Indicator & IKS Weather forecast \\
\hline 1. Risk & IKS is a guide to forming solutions related \\
& to livelihood activities. \\
& IKS information system in Mukonchi has \\
& not been particularly strong as a guide to \\
& preparation for farming and other related \\
& activities as well as in time of response. It is \\
& therefore not easy to mobilise the people.
\end{tabular}

2. Technical monitoring and warning services

\section{Communication and dissemination of warnings}

\section{Community response capability}

The IKS's experience is shrouded in oral tradition. Communication of the IKS is by the word of mouth which tends to affect the accuracy and consistency of information especially the warning services.

Has the potential for early warning system but there is need for better use of methods.

IKS employs oral communication whose frequency and preciseness cannot be ascertained.

IKS adopts new methods of communication so as to support information reaching the intended beneficiaries to prepare adequately. This can improve resilience among communities. IKS lacks a formalised data base.

IKS is an accumulation of significant knowledge about the weather events which should provide better planning and dependability and avoidance of disaster risks.

The grassroots have the knowledge and therefore can easily be mobilised to appreciate the range of risks and encourage them to prepare for action by taking the warnings seriously.

This bottom up approach is a tremendous advantage of IKS that can be exploited in disaster risk reduction and can be integrated into disaster risk reduction efforts at local level.
Conventional weather forecast

The conventional weather forecasts carry more specific information and possible risk assessment. It is possible to plan as a community and develop a measure of understanding of risks and how to reduce the impact of the disaster. There is more precise information here that would prepare a response in a manner that would reduce the risk and save the lives and livelihoods especially the socio-economic development.

The conventional system for technical monitoring and warning services provides more accurate information on multi hazards with clarity on procedures, institutional arrangements and communication networks. There is tremendous potential for early warning system with the likelihood to substantially reduce the risk of exposure to disasters

The purpose of warning is to reach those at risk so that they can prepare adequately for the eventualities.

For conventional warning system, the information can be easily transmitted but should be put in local vernacular for consumption by the local people. Usually, there are certain difficulties in presenting actual information.

However, there is need to develop easily accessible materials for use

The emphasis is on the involvement of local community. This is quite similar with the IKS but the difference being that knowledge for IKS is embedded in the community whereas the knowledge for the conventional system runs from top down and therefore from outside. Knowledge must be embedded in the communities so that people become the custodians of the knowledge infrastructure. It provides daily updates of weather reports that should reach the remotest parts of the rural areas and in a language that is not familiar. 
This study also sought to compare the IKS and the conventional weather forecasting systems by examining four keys as provided by the: Early Warning Conference (EWC) 111 Third International Conference on Early Warning, 27-29 March 2006, Born, Germany, International Strategy ISDR for Disaster Risk.

a) Risk knowledge

b) Technical monitoring and warning services

c) Communication and dissemination of warnings and

d) Community response capabilities

Despite the similarities and differences of the systems, there must be an effort to integrate the two for the benefit of the people (Table 3). This will greatly improve, among other things, resilience of African agriculture to climate shocks. However, climate forecasts can help farmers reduce their vulnerability to drought and weather extremes, while also allowing them to maximize opportunities when favourable rainfall conditions are predicted. Recent advances in climate modelling have resulted in increased ability to predict rainfall in many parts of the world with a lead time ranging from a few days to a few months, by using dynamical forecasts or statistical methods. Though a considerable amount of weather and climate information is now available to farmers, efforts must be made to make available emerging information to farmers for their decision-making. But caution must be made here that major improvements in weather forecasting that now exist should not be regarded as a panacea for weather related problems. Communities must continue to improve their well beings through the use of both IKS and conventional weather systems.

According to Hansen (2002) 'There are prerequisites for potential benefits of meteorological and climatological forecasts if they are to be realised by farmers. Firstly, these types of forecasts have to address a need for farmers that is real and perceived. Climate and weather forecasts have to be a relevant component of the climate or weather system at an appropriate farming spatiotemporal scale. Importantly, benefit also depends on the existence of decision options for the farmer which are sensitive to the particular incremental information that the forecasts provide and which are compatible with the farmer's goals.'

The recent Indonesia's Tsunami and the earthquake provide variable lessons that must be taken seriously by those talking about the weather and prediction. While the scientific systems are the best methods to forecasting the weather it is important to realise that the IKS should not be relegated to the background. We all know that the modern equipment to predict the phenomena were present on the island of Sulawesi consisting of Seismographic sensors, buoys, tidal gauges and GPS but the system could not predict the tsunami and the earthquake in time for action (www.Nytimes.com/interactive/2018/10/103/world/Asia/Indonesia). This gives a clear testimony about the importance of IKS which is embedded in culture, known by all and can easily be applied whenever necessary. People in the area know when the rain comes, what type, from where etc. Traditional methods for weather forecasting can be utilised for the purposes of short term and long term seasonal weather predictions by local communities. These methods according to the study range from biological, geographical and meteorological.

The need for appropriate interpretation cannot be overemphasized where farmers require a capacity to interpret correctly relevant forecasts which also have to be made with sufficient lead-time to make an impact on their decisions. Additionally, prediction of the relevant components of weather or climate variability is needed for relevant periods, at an appropriate scale, with sufficient accuracy and lead time, in a form that can be applied to the farmer's decision problems (Hasen, 2002).

In regions where there has been successful uptake of more complex climate and weather information by farmers, it has been important for farmers to participate in the development of appropriate response strategies to climate and weather information, especially about decisionsthat relate to climate forecast information suited for them. In this respect, farmers may be suspicious of a forecast system which they do not understand or have some ownership of the methods used to develop it, especially if they see the forecast as conflicting with their local traditional indicators (Patt, 2002).

Climate and weather forecasts may have absolutely no value unless key management decisions are identified through close interaction with farmers. The farmers' key management decisions should be considered so that they can impactpolicies and programmes on climate change as well as weather forecasts (Hammer et al., 2001). This is more than anything else the issue of good governance and institutional arrangements supported by well-founded existential experience from grassroots. 


\section{Conclusion}

Based on the findings, there is no doubt that the IKS is important and can still be employed in weather forecasting and superbly contribute to disaster risk reduction and savingof human life and property. But perhaps more importantly, efforts should be made to integrate IKS into the conventional weather forecasting system. There are those aspects of IKS which, to this day, remain relevant and critical to the weather forecasting system.

It is important therefore that local communities and their traditional leadership need to consolidate, document and utilise indigenous knowledge in weather prediction for planning their livelihood activities such as farming and fishing. The modern and traditional weather forecasters can work together to produce a comprehensive weather forecast that meets the needs of local communities. This study also noted that the younger generation need to be acquainted with ideas of IKS that are important in weather prediction from a traditional point of view. Thus indigenous knowledge on weather forecasting needs to be encouraged across all age groups. Perhaps, it needs to be part of our learning systems. Apart from making the young generation aware of the indigenous methods of weather forecasting, efforts must be made to make them aware and appreciate the need to safeguard natural resources such as forests, wildlife and water resources as critical elements of our heritage in the process of weather prediction.

\section{Conflict of interests}

The authors declare that there is no conflict of interests regarding the publication of this paper.

\section{References}

Ajibade et al. (2003). Indigenous approaches to weather forecasting in Asa LGA. Kwara State, Nigeria. African Journal of Indigenous Knowledge Systems, 2, 37-44.

Amanor et al. (1993). Cultivating Knowledge, Genetic Diversity, Farmers' Experimentation and Crop Research. London, Intermediate Technology Publications.

Boko et al. (2007). Africa Weather Change 2007, Impacts, Adaptation and Vulnerability. Contribution of Working Group II to the Fourth Assessment Report of the Intergovernmental Panel on Weather Change. Cambridge University Press.

Briggs, J. (2005). The use of Indigenous Knowledge in Development: Problems and Challenges. Progress in Development Studies, 5(2), 99-114.

Cruz, R. V. (2007). Climate Change, Impacts, Adaptation and Vulnerability. Contribution of Working Group II to the Fourth Assessment Report of the Intergovernmental Panel on Climate Change. Cambridge University Press, Cambridge, UK.

Goddard, L., \& Dilley, M. (2005). El Nino: Castrophe or opportunity, International Research Institute for Climate Prediction, The Earth Institute of Columbia, University Palisardes, New York. Journal of Climate, 18(5), 88-78.

Government Republic of Zambia. (2010). 2010 Census of Population and Housing: Central Statistics Office. Government Printer, Lusaka.

Hammer et al. (2001). Advances in application of climate prediction in agriculture. Agric. Syst., 70(2/3), 515-553.

Hansen, J. W. (2002). Applying seasonal climate prediction to agricultural production. Agric. Syst., 74(3), 305-307.

Makwara, E. C. (2013). However, not all waste should be discarded as some can be transformed into useful ... environment are not endangered in any way. European Journal of Sustainable Development, 2(1), 67-98.

Masahiro, N. (2017). Changes in the Soil Meso-and Micro-Fauna Community under the Impacts of Exotic Ambrosia Artemisiifolia. Retrieved from https://www.onelinelibrary-Wiley.com/journal/144.01703

Ngulube, P. (2017). Handbook of Research on Theoretical Perspectives on Indigenous Knowledge Systems in Developing Countries. Hershey, Pennsylvania.

Nindi, S. J., \& Mhando, D. G. (2011). Adaptations to Climate change and variability among smallholder farmers in Tanzania. In Filho, Chapter Ten in a Book titled Climate Change Management: Climate Change and the Sustainable Use of Water Resources. Springer Publication.

Patt, A. (2002). Effects of seasonal climate forecasts and participatory workshops among subsistence farmers in Zimbabwe. PNAS, 102, 12623-12628.

Phiri, A., Van Nikerk, D., \& Van Eede, S. E. (2016). Theoretical Orientation of Community based Disaster Risk Management. Global Journal of Human Social Scien, XVI(VI). 
Rautela, P., \& Karki, B. (2015). Weather Forecasting: Traditional Knowledge of the People of Uttarakhand Himalaya Department of Disaster Management, Disaster Mitigation and Management Centre, Uttarakhand Secretariat, Rajpur Road, Dehradun - 248 001, Uttarakhand, India.

Risiro et al. (2012). Emerging Trends in Educational Research and Policy Studies (JETERAPS), 3(4), $561-566$. Scholarlink Research Institute Journals, 2012.

Risiro, J., Mashoko, D., Tshuma, T., \& Rurinda, E. (2012). Weather Forecasting and Indigenous Knowledge Systems in Chimanimani District of Manicaland, Zimbabwe. Journal of Emerging Trends in Educational Research and Policy Studies, 3(4), 561-5661866.

Roncoli, C., Ingram, T. K., \& Kirshen, P. (2001). The Costs of and Risks of Coping with Drought: Livelihood Impacts and Farmers' Responses in Burkina Faso. Technical Report, Climate Forecasting and Agricultural Resources Project. University of Georgia, Athens.

Stenek, V., Boysen, B. C., Bohn, W., Evans, M., \& Tec, T. (2011). World Bank Group, International Finance Corporation. Climate Risk and Business Hydropower Kafue Gorge Lower Zambia. Washington DC. Retrieved from http://www.ifc/climatechange

WMO. (2013). GFCS-Intergovernmental Board on Climate Services. Special Bulletin 62. Retrieved from http://www.Nytimes.com/interactive/2018/10/103/world/Asia/Indonesia

\section{Copyrights}

Copyright for this article is retained by the author(s), with first publication rights granted to the journal.

This is an open-access article distributed under the terms and conditions of the Creative Commons Attribution license (http://creativecommons.org/licenses/by/4.0/). 